\title{
Pulsed EPR Methods to Study Biomolecular Interactions
}

\author{
Irina Ritsch ${ }^{\S a}$, Daniel Klose ${ }^{a}$, Henrik Hintz ${ }^{\mathrm{b}}$, Adelheid Godt ${ }^{\mathrm{b}}$, Gunnar Jeschke ${ }^{\mathrm{a}}$, and Maxim Yulikova
}

§SCS-Metrohm Award for best oral presentation in Physical Chemistry

\begin{abstract}
Orthogonal site-directed spin labelling in combination with pulsed EPR spectroscopy is a powerful approach to study biomolecular interactions on a molecular level. Following a surge in pulse EPR method development, it is now possible to access distance distributions in the nanometre range in systems of complex composition. In this article we briefly outline the necessary considerations for measurements of distance distributions in macromolecular systems labelled with two or more different types of paramagnetic centres. We illustrate the approach with two examples: an application of the Double Electron-Electron Resonance (DEER) method on a triple spin-labelled protein dimer labelled with nitroxide and $\mathrm{Gd}(\mathrm{III})$, and an optimisation study of the Relaxation Induced Dipolar Modulation Enhancement (RIDME) experiment for the orthogonal spin pair $\mathrm{Cu}($ (I)-nitroxide.
\end{abstract}

Keywords: DEER · Orthogonal spin labelling · Pulsed dipolar spectroscopy $\cdot$ RIDME · Site-directed spin labelling

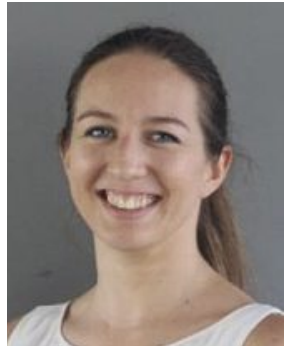

Irina Ritsch was born in Innsbruck, Austria. She completed her BSc at ETH Zurich in Interdisciplinary Sciences. As part of her Master's studies she spent six months on a research project at Trinity College in Dublin, Ireland. After she graduated in 2015 with an MSc at ETH Zurich, she joined the group of Professor Gunnar Jeschke at the Laboratory of Physical Chemistry as a PhD candidate. There she currently works on developing methods to study intrinsically disordered protein domains by electron paramagnetic resonance, including orthogonal spin labelling strategies.

\section{Using Paramagnetic Centres for Structural Biology}

Understanding the three-dimensional structure of biomolecules, or the arrangement of the components in complexes thereof, is an important step in discovering and explaining their biological function. Over the last few decades, Electron Paramagnetic Resonance (EPR) spectroscopic methods have become a valuable tool in the characterisation of biomolecular structure and interactions on a molecular level. ${ }^{[1,2]}$ EPR experiments are sensitive to paramagnetic centres in the sample, which may be part of an arbitrarily large biomolecule or biomolecular complex. These can be present either as native paramagnetic centres (such as biologically important metal ions, e.g. copper, iron or manganese), ${ }^{[3-10]}$ or as artificially introduced EPR-active centres. To the latter type belong paramagnetic centres that are covalently attached to the biomolecule of interest. They are, analogous to fluorescence labels, called 'spin labels' ${ }^{\text {' } 11-14]}$ and serve as a particularly useful source of information when the spin label is attached at a chosen site in the biomolecule. Such targeting of a particular molecular site (illustrated in Fig. 1(a)) is known as site-directed spin labelling (SDSL). A large body of work has been invested towards the development of SDSL protocols for peptides and proteins of nearly arbitrary size.[12,15-17]
In addition to SDSL of proteins, approaches are being developed to achieve universal, robust and size-independent SDSL methods also for oligonucleotides (DNA or RNA). ${ }^{[18-29]}$ The most popular choice for spin labelling of proteins is based on cysteine-targeting chemistry. The commercially available spin label MTSL (Fig. 1(a)) is one of the most prominent spin labelling reagents, among other reasons because the paramagnetic $\mathrm{N}$-oxyl-pyrroline moiety, which is linked to the cysteine via disulfide formation, is small and flexibly attached to the peptide backbone, which reduces the risk of perturbing the structure of the labelled molecule.

A major field of application for SDSL is related to the determination of distances between two spin labelled sites, which is possible in the low nanometre range $(1.5-10 \mathrm{~nm})$. With pulsed EPR methods we can isolate the magnetic dipole-dipole interaction, which depends on the inverse cube of the inter-spin distance, from other interactions. These methods in general are referred to as Pulsed Dipolar Spectroscopy (PDS). Along with the nitroxide-based spin labels other organic radical based (e.g. trityls $\left.{ }^{[32-35]}\right)$ spin labels and different types of metal ion-based ${ }^{[34-40]}$ spin labels have been developed and successfully tested in PDS experiments. In this article we illustrate why it is valuable to have a large toolkit of spin labels available for pulsed dipolar spectroscopy in a biological context. We particularly highlight the benefits that arise when different spin labels can be excited and detected independently, when they are thus said to be 'spectroscopically orthogonal', and we give two examples of EPR techniques that are applied for distance measurements with orthogonal spin labels.

\section{Extracting Distance Information from Pulsed Dipolar Spectroscopy Data}

The dipolar coupling frequency $v_{\mathrm{dd}}$ of two magnetic moments can be exploited for distance measurements. Assuming the usually well-fulfilled high-field and point-dipole approximations, it depends on the inverse cube of the distance $R$ between two spins, 


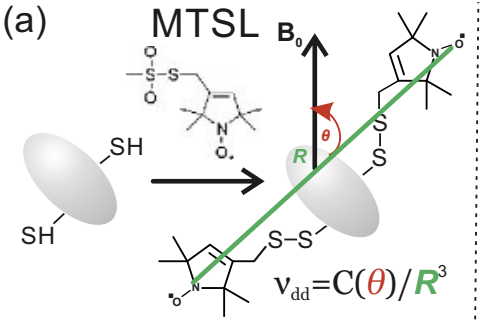

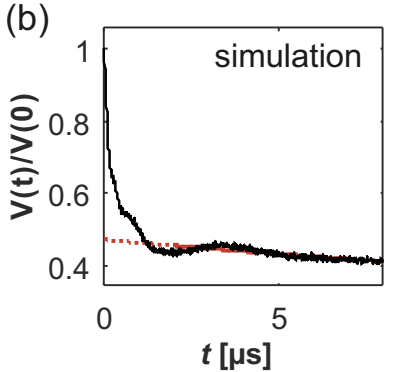
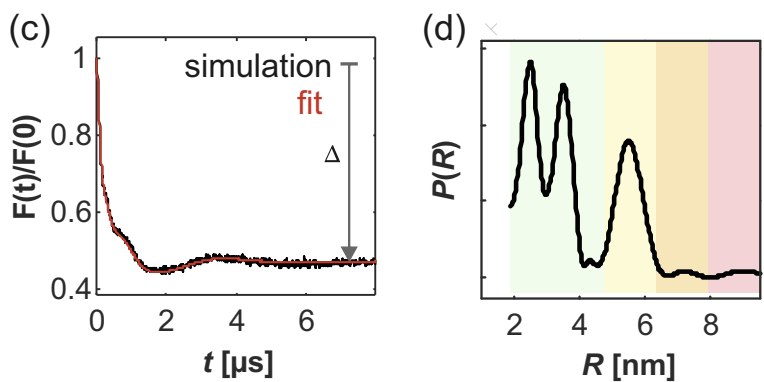

Fig. 1. (a) SDSL for distance measurement by pulsed EPR with the nitroxide spin label MTSL; (b) simulated time domain data as expected from a PDS experiment (black) calculated by powder averaging of Eqn. (1) with an input distance distribution and an assumed background function, which is re-fitted for data analysis (red); (c) the form factor (black) is obtained after division of the primary data by the background fit (the modulation depth $\Delta$ is indicated). It can be fitted for distance analysis (red). (d) Fitted distance distribution $P(R)$ obtained with model-free analysis of the form factor by Tikhonov regularisation (analysed with DeerAnalysis $2016^{[30,31]}$ ). The color-coding indicates the reliability of the distribution in different distance ranges as a function of maximum dipolar evolution time (green: reliable shape, yellow: reliable mean distance and width, orange: reliable estimate of mean distance, red: no prediction possible for this dipolar evolution time).

as well as on the angle $\theta$ between the inter-spin vector and the external magnetic field $B_{0}$

$v_{\mathrm{dd}}(R, \theta)=\frac{\mu_{0} \mu_{B}^{2}}{4 \pi h} \frac{g_{A} g_{B}}{R^{3}}\left(1-3 \cos ^{2}(\theta)\right) \approx 52.04 \mathrm{MHz} \frac{\left(1-3 \cos ^{2}(\theta)\right)}{(R / \mathrm{nm})^{3}}$.

An illustration can be found in Fig. 1(a). For distance measurements between organic radicals, the pre-factors (magnetic permittivity of vacuum $\mu_{0}$, Bohr magneton $\mu_{\mathrm{B}}$, Planck's constant $h$, g-factors of the coupled spins $g_{\mathrm{A}}, g_{\mathrm{B}}$ ), are typically summarised into one constant that implicitly defines the range of $R$ accessible for distance measurements by PDS, which falls into the nanometre regime. Experimental methods to measure these dipolar couplings will be introduced in the next section, but in general the output of any PDS measurement is a time-dependent signal containing oscillations with a distribution of dipolar frequencies, which originates from the underlying intramolecular distribution of the spin-spin distances. The high sensitivity of PDS methods allows to work with spin concentrations in the low micromolar range, which is sufficiently dilute that we can treat each individual spin pair as an isolated system. Due to transverse relaxation of the detected spins, it is often difficult to resolve coupling frequencies smaller than $50 \mathrm{kHz}$, which correspond to a maximum accessible distance of $R_{\max } \approx 10 \mathrm{~nm}$, or a period of dipolar oscillations of about $20 \mu \mathrm{s}$. Because the average number of intermolecular spin pairs in a homogeneous solution increases with the square of the distance, these intermolecular dipole-dipole interactions are not fully negligible for distances up to several tens of nanometres, ${ }^{[41-43]}$ even though the coupling frequencies become very small. The lower limit of accessible distances $R_{\min }$ is given by the distance at which the strength of the electron spin-spin interaction is comparable to the excitation bandwidth of the microwave pulses. For commonly used EPR setups $R_{\min }$ is usually between 1.2 and $1.8 \mathrm{~nm}$. It may also happen that at short spin-spin distances, spin exchange interaction becomes relevant or even dominant, which strongly complicates the analysis of distance distributions. The angular dependence in Eqn. (1) implies that the orientation of a given spin pair with respect to the external magnetic field must be fixed on the time-scale of the experiment, in order to avoid time-averaging of the dipolar interaction. This is typically achieved by freezing the sample, but other methods to immobilise biomolecules for PDS are emerging, which even have enabled PDS at ambient temperature. ${ }^{[24,32,44]}$ The data analysis procedure to extract distance distributions from PDS data is illustrated in Fig. 1(b)-(d). It begins with the removal of the intermolecular distance distribution ('background' signal contribution). In order to convert the thus obtained 'form factor' to the distance domain, it is necessary to invert an integral equation of the Fredholm type, which is known to be an ill-posed problem. This can either be done by a model-based approach (e.g. fitting with a Gaussian distribution), or in a model-free fashion (e.g. using Tikhonov regularisation). ${ }^{[45,46]}$ Note that new approaches, for example based on neural networks ${ }^{[47]}$ or the simultaneous analysis of several PDS datasets, ${ }^{[48]}$ are emerging. All methods generate a distance distribution $P(R)$, which can then be used as a long-range restraint in structure determination of biomolecules, ${ }^{[15,20,49,50]}$ to monitor conformational changes and detect snapshots of molecule dynamics, ${ }^{[49,51-53]}$ or to study biomolecular interactions. ${ }^{[54,55]}$ In principle, no prior information on the mean distance and width or shape of an underlying distance distribution is required, provided sufficiently high data quality can be achieved. Benchmarking of various pulse EPR methods to measure dipolar couplings has been performed for example using model compounds with a well-defined and narrow distance distribution between two spins (often called 'rulers'), or with biomolecules with a known structure. ${ }^{[32,33,56]}$

\section{Pulse Sequences for Pulsed Dipolar Spectroscopy}

Several PDS pulse sequences were developed, which differ in the way the two spins of a dipole-dipole coupled pair are excited. In one set of techniques, ${ }^{[34,57-59]}$ both spins of a pair are excited by all pulses. Such techniques are more relevant for distance measurements with pairs of identical spin labels. We focus here on another type of PDS experiments, to which the Double ElectronElectron Resonance (DEER, also known as PELDOR) experiment, ${ }^{[60,61]}$ as well as the Relaxation Induced Dipolar Modulation Enhancement (RIDME) experiment, ${ }^{[62,63]}$ belong, where one of the spins in the pair is the observer (spin A) and the other one is flipped by pump excitation at a second frequency or, statistically, by relaxation (spin B). Most experiments with spectroscopically orthogonal spin labels are based on this principle.

The 4-pulse DEER pulse sequence (Fig. 2(a)) is currently the major workhorse of EPR experiments with nitroxide spin labels, and the RIDME pulse sequence (Fig. 2(b)) is becoming an attractive alternative for PDS experiments involving paramagnetic metal ions. In DEER, we need to place pulses at two different frequencies (pump and probe frequencies) within the combined EPR spectra of the two spin labels. The method works best when the pump and probe bandwidths together cover a substantial fraction of the EPR spectra. However, overlap of the microwave pulse bandwidths must be avoided because it leads to signal reduction and may introduce artefacts. ${ }^{[64-66]}$ This excitation band separation may be more easily achieved if the two EPR spectra of the pumped and the detected spin do not overlap. Experimental complications for the DEER experiment appear, however, when the frequency gap between the EPR spectra of the spins becomes too large. The limiting factors are excitation and detection bandwidths. As a guideline, with the newest broadband spectrometers, equipped with arbitrary waveform generators (AWGs), ${ }^{[67,68]}$ a pulse band- 
(a)

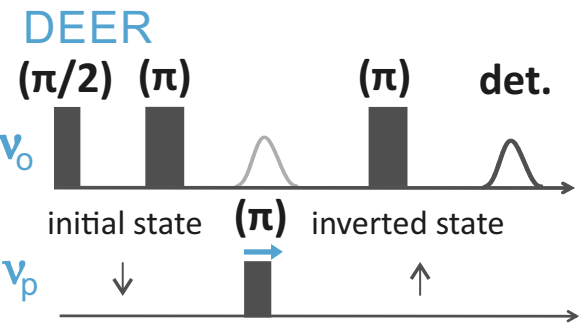

(b) RIDME

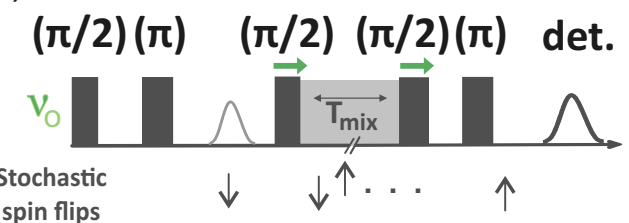

(all $\mathbf{v}$ ) initial state inverted state (c)
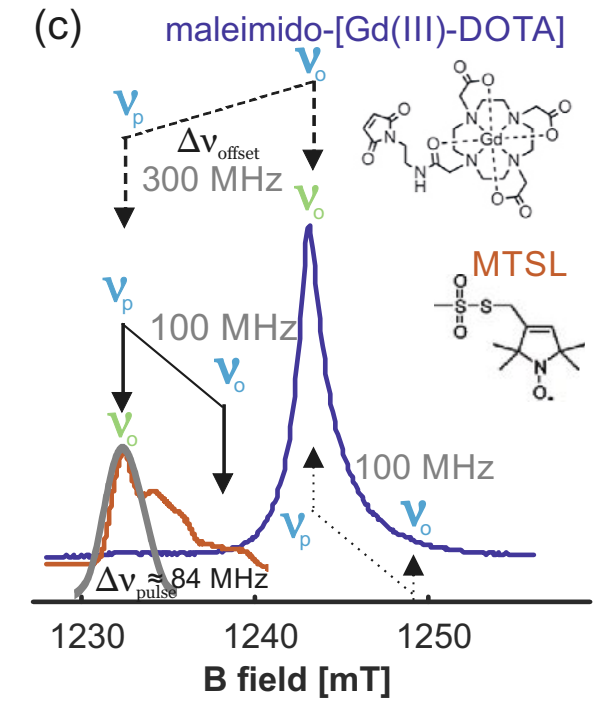

(d)

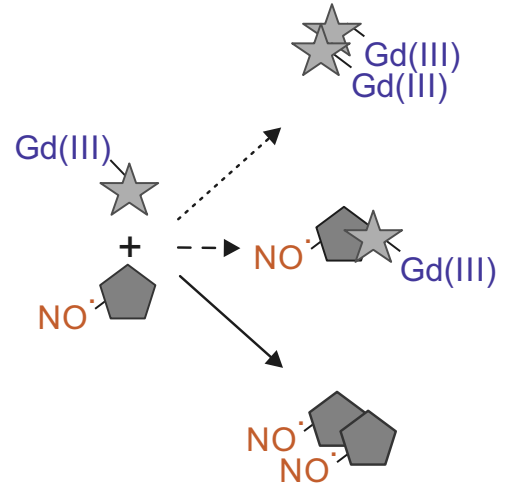

Fig. 2. (a) Pulse sequence for 4-pulse DEER, and (b) for RIDME; DEER is a two-frequency experiment ( $\left.V_{0}, V_{p}\right)$, the RIDME experiment uses pulses at only one frequency $(\mathrm{V})$. (c) Echo-detected EPR (EDEPR) spectra of MTSL (orange) and maleimido-[Gd(III)-DOTA] (blue) at Q-band, and approximate excitation band of a rectangular pulse (grey). Typical pump $\left(V_{p}\right)$ and observation $\left(V_{\circ}\right.$, resp. $\left.V_{0}\right)$ positions for DEER and RIDME are indicated. The three shown DEER setups are optimized to detect nitroxide-nitroxide (solid arrows), nitroxide-Gd(II) (dashed arrows), resp. Gd(III)-Gd(ıI) (dotted arrows) dipolar couplings. (d) In a sample where two potentially interacting components are labelled with spectroscopically orthogonal spin labels we can distinguish different interaction combinations by performing selective PDS experiments (compare solid/dashed/dotted arrows in Panel (c)).

width of about $1.5 \mathrm{GHz}$ can be achieved, ${ }^{[69]}$ which is more than ten times larger than the bandwidth of rectangular microwave pulses in the previous generation of spectrometers $(\sim 100 \mathrm{MHz})$. The latter is easily sufficient for nitroxide-nitroxide distance measurements, but it is too narrow-banded for efficient excitation of spin labels based on metal ion centres, which have significantly broader EPR spectra.

The RIDME experiment exploits a different principle for achieving inversion of the B spin, which is particularly promising for the combination of organic radicals with paramagnetic metal ion centres. The non-equilibrium magnetization of the A spins is stored along the direction of the static magnetic field for a fixed part of the pulse sequence. During this period, the B spins flip stochastically with a certain probability, depending on their longitudinal relaxation time. These spin flips invert the magnetic field induced by spin B at the site of spin A and thus shift the A spin resonance frequency by the dipole-dipole interaction. This effect can be detected after transferring the magnetization of the A spins back to the transverse plane to form a spin echo. RIDME requires pulses at only one microwave frequency, and B spin inversion is thus not limited by the bandwidth of an additional pump pulse.

\section{Spectroscopically Orthogonal Spin Labels in Pulsed Dipolar Spectroscopy}

Spin labels are considered spectroscopically 'orthogonal' if the intrinsic spectral differences between the paramagnetic centres can be exploited for selective excitation. ${ }^{[70,71]}$ Over the last decade there were many important advances in the development of PDS experiments with pairs of spectroscopically orthogonal spin labels. Metal ion-nitroxide spin pairs were among the first investigated combinations. ${ }^{[72]}$ For DEER experiments on such systems it is suggested to pump the nitroxide spin and to detect the metal ion spin. This is favourable in most situations in terms of longitudinal relaxation properties and maximises the fraction of inverted spins. Broad-band excitation schemes can strongly increase the sensitivity. ${ }^{[73,74]} \mathrm{Gd}(\mathrm{III})$-nitroxide DEER was early recognised as a suitable technique for spectroscopic selection (compare Fig. 2(c)). ${ }^{[75-77]}$ The pulse setup optimization takes into account that unwanted excitation of the detected metal ion spins by the pump pulse on the nitroxide can lead to partial suppression of the detected echo. ${ }^{[36,70,77]}$ Also $\mathrm{Cu}(\mathrm{II})-\mathrm{Cu}(\mathrm{II})^{[78]}$ and $\mathrm{Cu}(\mathrm{II})-$ ni- troxide ${ }^{[72]}$ DEER were developed. It was found that 'orientation selection' can strongly interfere with data interpretation when using stiff molecular rulers. This arises because the kernel-based data analysis usually assumes a uniform distribution of spin-pair orientations contributing to the data (included as an effective averaging over the dipolar angle $\theta$ ), and we observe artefacts if this is not fulfilled due to experimental reasons. ${ }^{[58,72,78-81]}$ Several studies were dedicated to compensating for this effect. ${ }^{[82-84]}$ Considerable effort was also invested in the enhancement of detection sensitivity for metal ion-based spin labels. This includes a spin pre-polarization technique for PDS experiments with detection on high spin metal ions. ${ }^{[73]}$ Furthermore, the possibility to use the RIDME technique for detecting distance distributions between high-spin metal centres was shown. ${ }^{[85-87]}$ In RIDME with high spin centres, higher orders of spin inversion (with an effective total change of magnetic quantum number $\Delta \mathrm{m}_{\mathrm{s}}>1$ ) can occur. Such dipolar evolution data can still be analysed in terms of distance distribution by applying a slight modification to the kernel function, as was demonstrated with a calibration for Gd(III) and Mn(II)-based spin labels. ${ }^{[85,86]}$ RIDME measurements on metal ion-nitroxide spin pairs were reported, where, in contrast to DEER spectroscopy, the optimal detection position is on the nitroxide spin.[6,79,80,82,88] This is dictated by the relaxation properties of metal ion and nitroxide spins, but typically also favourable in terms of transverse relaxation and reduced orientation selection.

\section{Using Spectroscopically Orthogonal Spin Labels to Resolve Ambiguous Distance Information}

One of the strengths of PDS spectroscopy is that distances can be determined in biomolecules that are labelled with the same type of spin label at two sites. However, identical labels can also be a disadvantage, if the biomolecules aggregate unspecifically or if (controlled) aggregation is the focus of the study. Whenever many spin-labelled molecules are in close proximity (within the sensitive range of DEER of $~ 1.5-10 \mathrm{~nm}$ ), short-range intermolecular distance distribution peaks appear, which cannot be distinguished directly from intramolecular distance peaks. This means that tedious control experiments using singly-labelled proteins, and/or spin dilution experiments are required to disentangle the underlying intra-molecular distance distributions. In a spin dilution experiment one mixes unlabelled protein with (double-) 
spin-labelled protein at a known ratio. ${ }^{[89]}$ We thereby reduce the number of protein complexes in which more than two spin labels are present, as long as the unlabelled and the labelled proteins mix homogeneously. Consequently, the intensity of the intermolecular distance distribution peaks is reduced. This is a difficult task if the intramolecular distance distribution is broad and/or overlaps significantly with the intermolecular distance distribution. The required degree of spin dilution has to be determined case by case. For aggregating material it may be necessary to use very low ratios of labelled to unlabelled biomolecules, which reduces sensitivity, and while spin dilution removes intermolecular artefact peaks from the distance distribution, it cannot be directly used to monitor the aggregation state of the sample.

An elegant possibility to monitor the aggregation state is to use spectroscopically orthogonal spin labels. These are either attached at different sites of one biomolecule, or, if the study involves more than one type of biomolecule in a complex, each type of biomolecule is labelled with a specific spin label spectroscopically distinguishable from the other spin labels. In either case we can determine the aggregation state of the sample by performing a PDS experiment which will reveal a distance distribution if the biomolecules interact with each other, as is illustrated in Fig. 2(d). ${ }^{[71,90]}$ In such experiments we not only see if there are intermolecular interactions present at all, but we also get distance information associated with the interaction. SDSL with orthogonal spin labels is very powerful, when two components (e.g. components X and $\mathrm{Y}$ ) of a complex interact with each other, but also interact with themselves. PDS with only one type of spin label could not distinguish the two situations, but if the components are labelled with spectroscopically orthogonal spin labels we can differentiate all three possible combinations, $\mathrm{X}-\mathrm{X}, \mathrm{X}-\mathrm{Y}, \mathrm{Y}-\mathrm{Y}$, in a sample. Clearly, selective excitation of the two species must be sufficiently good to have this benefit. The appropriate choice of spin label combination, however, mostly depends on the biological question at hand. Some metal ion complexes, for example Gd(III) complexes or trityls, are more stable than common nitroxide-based spin labels in reducing environments, which are encountered for example in the cellular cytoplasm in in cell experiments. ${ }^{[40,91-95]}$ Active research is on-going to develop more stable nitroxides for such applications. ${ }^{[16,96-100]}$ Other considerations are hydrophobicity, charge state or bulkiness of the spin label, all of which can disrupt protein folds, or inhibit interactions if chosen unsuitably.

\section{Selective Excitation of Different Types of Spin Centres}

To illustrate selective excitation, which is the prerequisite for spins to be considered spectroscopically orthogonal, we show the
EPR spectra of four different EPR-active species (a nitroxide, resp. $\mathrm{Cu}(\mathrm{II})-, \mathrm{Gd}(\mathrm{III})-$, or $\mathrm{Mn}(\mathrm{II})$-based metal ion complexes) in Fig. 3(a-c). As can be seen, the spectral shape and width of the EPR spectra are very diverse. In a biomolecule or biomolecular complex with a set of different spin labels attached at different sites, we can selectively excite the two types of electron spins by choosing the appropriate resonance frequency and microwave pulse power, or by their relaxation properties. ${ }^{[70,71,101]}$ Not surprisingly, very good spectroscopic selection can be achieved if the EPR spectra of the two spin labels do not overlap. However, even if there is partial overlap we observe in general that the longitudinal relaxation of metal ion complexes is much faster than that of organic radicals at any given temperature. This can be exploited to achieve suppression of the signal from a simultaneously present organic radical (such as a nitroxide) by choosing very fast experiment repetition rates. The slowly relaxing spins are then saturated and thus only weakly observed (compare Fig. 3(b,c)) Such intentional saturation of spin transitions, as well as other spectroscopic filtering methods based on differences in longitudinal relaxation can be used in combination with many pulsed EPR experiments. ${ }^{71,90,102]}$ Transverse relaxation properties between paramagnetic species differ as well, but the differences are typically much smaller than for longitudinal relaxation. Paramagnetic species can also be separated by the total spin quantum number $\mathrm{S}$. Spin transitions of high-spin paramagnetic centres $(\mathrm{S}>1 / 2)$ are characterized by a larger transition moment and thus require less microwave power to be excited, as compared to the case of lowspin paramagnetic centres. The optimal power for inversion can be, for example, determined by a nutation experiment (see Fig. $3(d)$ ), in which the duration of an inversion pulse is incremented at fixed microwave pulse power. Such pulse optimisations are useful when selectively exciting high-spin metal ion-based spin labels (often $\mathrm{Gd}(\mathrm{III})$ or $\mathrm{Mn}(\mathrm{II})$ ) in the presence of nitroxides.[101]

\section{The Rpo4-Rpo7 Complex as a Model System}

In this section, we demonstrate the application of PDS with spectroscopically orthogonal nitroxide and $\mathrm{Gd}(\mathrm{III})$-based spin labels, to identify intra-subunit and inter-subunit distance distributions in a protein complex. We use the complex between subunits Rpo4 and Rpo7 (formerly known as subunits F and E) of the Archaeal RNA polymerase of M. jannaschii as a model system, which is well characterised by other structural and biochemical methods, ${ }^{[103-106]}$ and it was found to be stable and fairly rigid. ${ }^{[107]}$ Previous studies on this model system include an early contribution to the in silico modelling of DEER and fluorescence data, ${ }^{[107]}$ and the first experimental demonstration of a PDS correlation experiment between three nitroxide spins in terms of a distance (a)

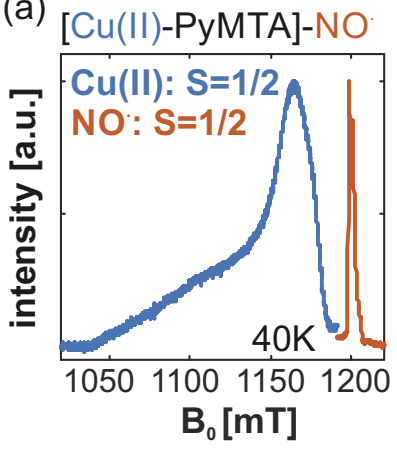

(b)

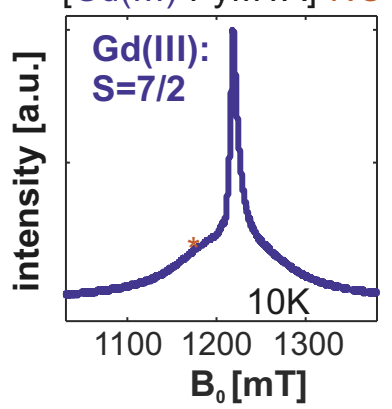

(c)

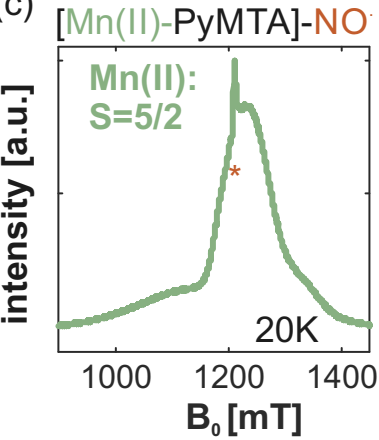

(d)

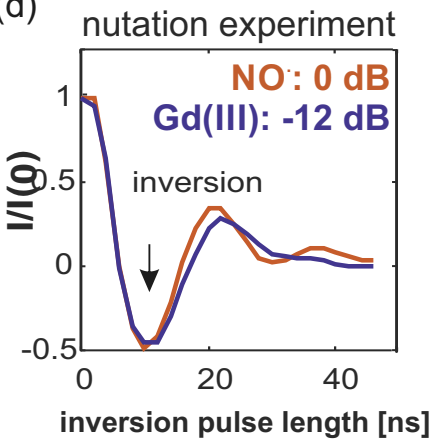

Fig. 3. (a-c) EDEPR spectra of frozen samples of paramagnetic centers; (a) the spectra of [Cu(॥)-PyMTA] (dark blue) and nitroxide (orange) are spectrally separated. (b) The spectra of [Gd(III)-PyMTA] (blue) and nitroxide (expected at the position of the orange star) would overlap, but the nitroxide is suppressed with the chosen pulse settings (shot repetition rate: $100 \mu$ s, pulse power optimized for Gd(II)). (c) Suppression of the nitroxide can also be achieved with respect to [Mn(II)-PyMTA] (green), but a weak residual intensity in the Mn(II) spectrum is still observed (indicated by orange star). (d) Nutation profiles measured on the maxima of $\mathrm{Gd}(\mathrm{II})$ (dark blue, measured with a pulse attenuation of $12 \mathrm{~dB}$ ), and nitroxide (orange, $0 \mathrm{~dB}$ ). The same nutation profile for Gd(III) (high spin) and nitroxide (low spin) is observed because the pulse power for the Gd(III) measurement was reduced by a factor of four w.r.t. the nitroxide measurement. 
(a)

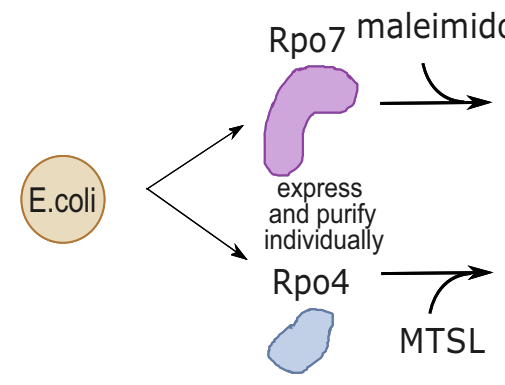

(b)

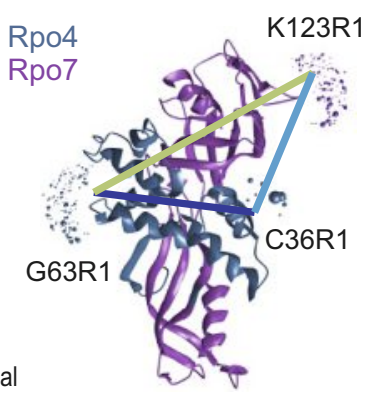

(c)

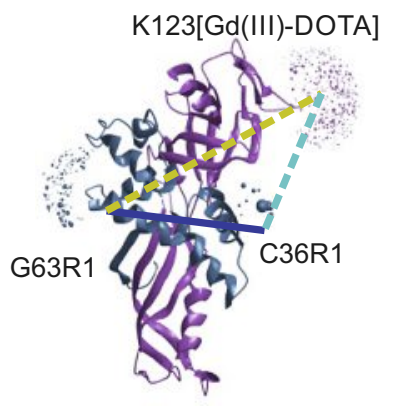

Fig. 4. (a) Scheme for SDSL sample preparation of the Rpo4/7 complex. The subunits can be conveniently labelled individually with spectroscopically orthogonal spin labels prior to complex formation. (b) Visualization of rotamers probability distribution 'clouds' generated with MMM ${ }^{[31]}$ of MTSL simulated at $298 \mathrm{~K}$ attached to residues 36 and 63 in Rpo4 (purple), resp. residue 123 in Rpo7 (blue) in the Rpo4/7 complex (pdb: 1GO3 ${ }^{[102]}$ ).

correlation map using the Triple Electron Resonance (TRIER) experiment. ${ }^{[108,109]}$ Our labelling strategy for this study is shown in Fig. 4(a). Both cysteine mutated subunits were spin labelled individually in the fully denatured state (in buffer with $6 \mathrm{M}$ urea), which alleviated any accessibility issues, and typically resulted in very high labelling efficiencies. Complex formation was achieved by a refolding step. In Fig. 4 we show the X-ray crystallographic structure ${ }^{[106]}$ of the Rpo4/7 complex that was in silico modified to carry spin labels (e.g. MTSL-labelled $(=\mathrm{R} 1)$ at positions Rpo7: K123R1, and Rpo4: C36R1, G63R1). The probability distribution of the location of the electron spin relative to the protein backbone is calculated using a rotamer library approach ${ }^{[110]}$ and visualised as coloured clouds. ${ }^{[110]}$ The corresponding three possible simulated spin-spin distance distributions (Rpo7: K123R1 / Rpo4: C36R1, Rpo7: K123R1 / Rpo4: G63R1, and Rpo4: C36R1 / Rpo4: G63R1) are well-separated (see Fig. 5(a)), and spread over a large range of distances $(2-6 \mathrm{~nm})$.

The nitroxide-nitroxide DEER experiment on the protein complex labelled with MTSL at all three sites was performed on a frozen sample measured at a temperature of $50 \mathrm{~K}$ at Q-band frequencies (34 GHz) (Fig. 5(b)). The distance analysis reveals three resolved distance peaks (Fig. 5(c)). From this experiment alone, however, it is impossible to tell which of the three peaks corresponds to the Rpo4-internal distance (Rpo4: C36R1-G63R1) and which peaks correspond to the inter-subunit distances (Rpo4:C36R1, resp. G63R1 - Rpo7: K123R1).

Because we are dealing with a three-spin system, and because the intramolecular dipolar evolution signal in DEER is a product of contributions from all possible pair-wise spin combinations, additional sum and difference frequency terms of the dipolar coupling frequencies appear. ${ }^{[111,112]}$ These terms lead to so-called 'ghost peaks' in the distance distribution. Computational data analysis approaches to reduce the intensities of such ghost peaks have been introduced. ${ }^{[111]}$ Experimentally, we can suppress the sum and difference terms because they depend quadratically on the pump pulse inversion efficiency. ${ }^{[111,112]}$ Accordingly, using an attenuated pump pulse we suppress multi-spin effects and the shortest and the longest distance peaks observed in the triple MTSL-labelled sample are shifted to longer mean distances. The relative intensities of the two components in the central distance peak are also affected. Unfortunately, this also leads to a substantial reduction of the modulation depth and hence of the signal-to-noise ratio. One could also reduce multi-spin effects by 'spin dilution', which could simultaneously help to resolve the peak assignment issue, again at the cost of signal-to-noise ratio. ${ }^{[89]}$ Exchanging one of the nitroxides for a Gd(III)-based spin label, which is spectroscopically orthogonal as discussed above, can solve this problem in an elegant and reliable way, as has already been demonstrated in earlier works. ${ }^{[51,55,75,113,114]}$ Here, the subunit Rpo4 with two labelling sites (C36, G63C) was labelled with MTSL and the subunit
Rpo7 with one labelling site (K123C) was labelled with maleimido-[Gd(III)-DOTA]. The PDS data and distance distributions of this sample with mixed spin labels are shown in Fig. 5(d-f). With the nitroxide-nitroxide DEER experiment we find only one peak, centred at $\mathrm{R}_{\mathrm{NO}-\mathrm{NO}}=4.5 \mathrm{~nm}$ which corresponds to the central peak in the distance distribution obtained with the triple MTSL-labelled sample. We have thus identified this peak as the intra-subunit distance within Rpo4. In an application study this distance could be used as a reporter distance that monitors whether the subunit undergoes some structural rearrangement upon complex formation or substrate binding (distance before complex formation not shown here). The complementary DEER experiment to measure inter-subunit distances between nitroxide and $\mathrm{Gd}(\mathrm{III})$ was performed at $10 \mathrm{~K}$ (instead of $50 \mathrm{~K}$ for nitroxide-nitroxide DEER), with different pump and detection positions, ${ }^{[39,95]}$ illustrated in Fig. 2(c). As expected, we find two peaks in the distance distribution, at $\mathrm{R}_{\mathrm{Gd}(\mathrm{III})-\mathrm{NO}, 1}=3.5 \mathrm{~nm}$ and $\mathrm{R}_{\mathrm{Gd}(\mathrm{III})-\mathrm{NO}, 2}=6-7 \mathrm{~nm}$. The overlay of the distance distributions obtained with the two samples is shown as well. We clearly see that all three peaks found for the triple MTSL-labelled sample were also detected in the combined results from the samples with the combination of nitroxide and Gd(III)based spin labels. We furthermore observe that the best agreement is found with the trace that was measured with the suppression of ghost peaks. For the nitroxide-nitroxide DEER, ghost peaks are absent because we only have two nitroxide moieties per protein complex. Contaminations due to Gd(III) excitation by detection and pump pulses are in principle possible, but they are very weak in nitroxide-nitroxide DEER due to (a) the fast relaxation of $\mathrm{Gd}(\mathrm{III})$ at $50 \mathrm{~K}$, and (b) the strong difference in the microwave power required for the excitation of nitroxides and Gd(III) centres. For the case of Gd(III)-nitroxide DEER there are still ghost peaks present because two different Gd(III)-nitroxide pairs contribute per protein complex. However, the number of combination frequencies is reduced, from six for the triple MTSL-labelled sample to two for Gd(III)-double MTSL-labelled sample. Furthermore, in the given experiment the two detected real distance peaks have very different mean distances, and thus a very strong difference in the corresponding dipolar frequencies. As a result, the two ghost peaks nearly coincide with the short distance peak (around $3 \mathrm{~nm}$ ), which corresponds to about eight times larger dipolar frequency than the longer-distance peak (around $6 \mathrm{~nm}$ ). Any type of selective Gd(III)-Gd(III) PDS experiment (e.g. Gd(III)-Gd(III) RIDME or DEER) would complete the set of available PDS measurements in the orthogonally spin labelled sample, and provide information on possible aggregation of Rpo4/7 complexes. In our example, we did not expect such aggregation and skipped this step. In the present case, we would expect to observe a smooth intermolecular decay in the Gd(III)-Gd(III) PDS experiment, where the decay rate depends on the volume concentration of protein dimers and on the particular settings of the PDS experiment. 
(a) MMM simulation

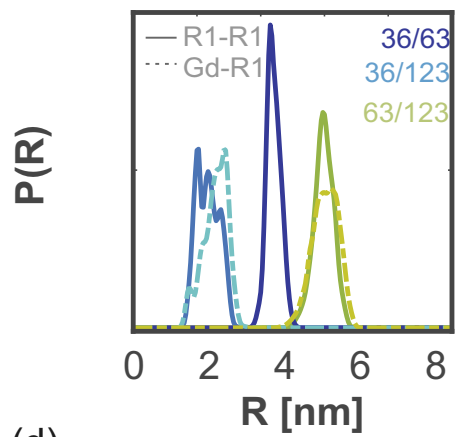

(d)

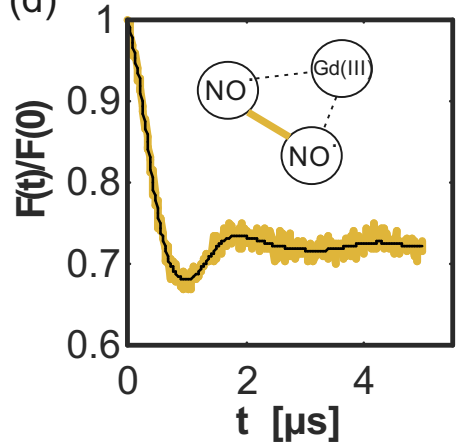

(b)

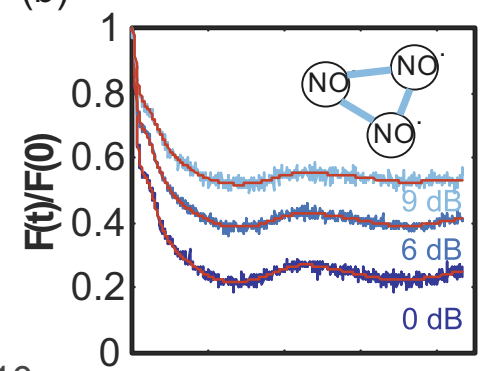

10

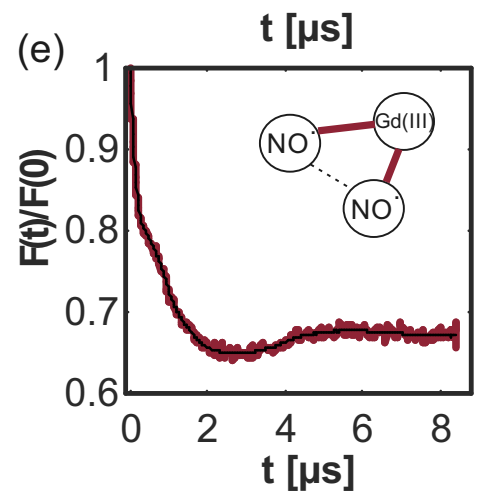

(c)

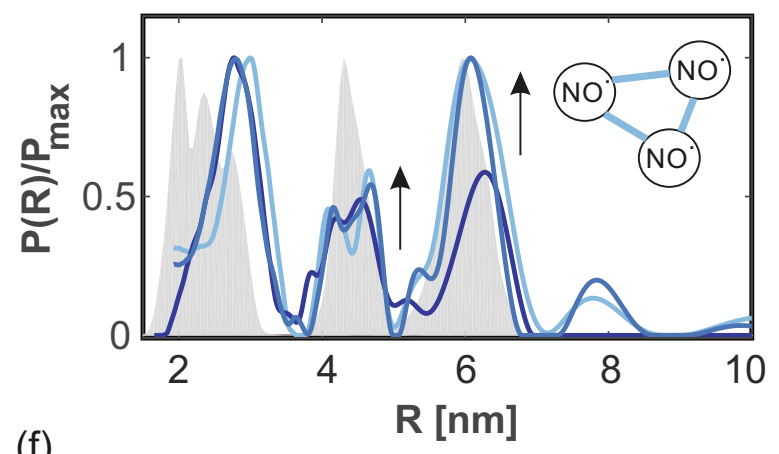

(f)

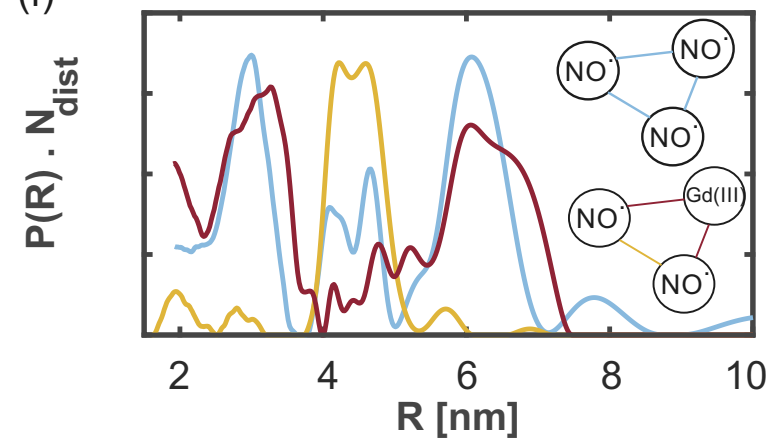

Fig. 5. (a) Simulated distance distributions between the chosen labelling sites (R1=labelled with MTSL, Gd=labelled with maleimido-[Gd(III)-DOTA]). (b) Experimental form factors and fits (red) obtained with nitroxide-nitroxide DEER with full inversion pulse power (dark blue) and attenuated inversion pulse powers (middle and light blue, relative attenuation of inversion pulses in dB) measured on the triple MTSL-labelled complex of Rpo4 (36R1, 63R1) /Rpo7 (K123R1); (c) Fitted distance distributions and expectation from simulation (grey area) obtained from the nitroxide-nitroxide DEER in panel (b). (d) Experimental form factor (yellow) and fit (black) from nitroxide-nitroxide DEER in the triple labelled protein complex with spectroscopically orthogonal spin labels (Rpo4 (36R1, 63R1) /Rpo7 (K123[Gd(III)-DOTA])). (e) Experimental form factor (red) and fit (black) from nitroxide-Gd(III) DEER obtained with the same sample; (f) distance distributions obtained with the spectroscopically orthogonal spin-label protein complex (yellow: nitroxide-nitroxide, red: nitroxide-Gd(III)); the distance distribution from the sample labelled only with MTSL is overlaid (blue).

\section{Water-Soluble Molecular Rulers for the Optimisation of $\mathrm{Cu}(\mathrm{II})$-Nitroxide RIDME}

In the previous example we demonstrated that PDS measurements with pairs of spectroscopically orthogonal spin labels are particularly useful in the study of intermolecular interactions. It is often possible to determine complex formation equilibria, because they can be related to the amplitude of the dipolar modulations (modulation depth). ${ }^{[51,115]}$ It is not rare, however, that in the studies of association/dissociation equilibria one of the constituents is present in excess. There is thus a demand for complementary PDS methods, which offer detection on either of the spectroscopically orthogonal spins. For metal ion-nitroxide pairs, RIDME (detected on the nitroxide) and DEER (detected on the metal ion spin) are such complementary methods. Note that in RIDME the inversion of the spins is bandwidth-independent, since it happens due to the spontaneous spin flips, while in DEER the inversion is performed by a (broadband) microwave pulse. In the last three years there were several publications, proposing different applications for metal ion-nitroxide RIDME. ${ }^{[84,88,115]}$ Water-soluble rulers with $\mathrm{Cu}$ (II)-PyMTA and $\mathrm{Cu}(\mathrm{II})$-TAHA moieties as the metal ion based spin labels, and the same nitroxide moiety as contained in MTSL, can be used for the optimisation of the RIDME technique. In Fig. 6 we show the structures of the two types of $\mathrm{Cu}(\mathrm{II})$-nitroxide rulers, and the PDS data for the one with $\mathrm{Cu}$ (II)-TAHA. The synthesis of the molecular rulers was performed in a modular way which allows to tune the intramolecular spin distance by the linking of a defined number of repeating units to obtain the spacer, and to easily vary the metal ion ligand. The good solubility in aqueous solvent was achieved by PEGylating the spacer backbone, and allows us to study the performance of RIDME at conditions similar to those typically used in biological applications. The narrow spin-spin distance distributions obtained with such molecular rulers allow for a detailed evaluation of the spectroscopic method, as well as the identification of sources of artefacts. The accurate treatment of intermolecular background decay, echo-crossing artefacts, and electron-nuclear modulation artefacts were studied, which should allow broad application of the RIDME technique. It was found that main features of the $\mathrm{Cu}$ (II)-nitroxide RIDME are very similar for samples in organic solvents and in water/glycerol mixtures. This allows to use the relatively large set of $\mathrm{Cu}$ (II)nitroxide RIDME data obtained from samples in organic solvents for optimization of biomolecular structural studies, which are performed in water-based buffer solutions mixed with a cryo-protectant, like glycerol, ethylene glycol, or sucrose.

In addition, it is possible to perform the RIDME pulse sequence with frequency-swept pulses that cover the entire spectrum of the nitroxide spins (bandwidths of 350-450 MHz, supplied by an AWG). Fourier transform of the spin echo shape at every combination of inter-pulse delays then allows adding a 'direct' dimension to the RIDME experiment, in addition to the 'indirect' dimension, originating from the inter-pulse delay variations. This allows to construct 2D plots with EPR-correlated dipolar spectra, useful in the analysis of orientation selection in stiff spin labelled molecules. At the same time, such a 'chirp-RIDME' technique is useful for averaging the angular dependence of the dipolar interaction, because we achieve orientation averaging both for the nitroxide and the $\mathrm{Cu}(\mathrm{II})$ spins in a single experiment.

\section{Conclusion and Outlook}

We summarised here pulse EPR approaches to study interactions in biomolecular complexes and aggregates, which depend on spectroscopic selection of different paramagnetic centres. The application of such approaches to biological systems of virtually arbitrary size is a powerful method for the study of complex multi-component systems, especially if the individual components of such a system can be orthogonally spin-labelled. The use of mod- 
(a)

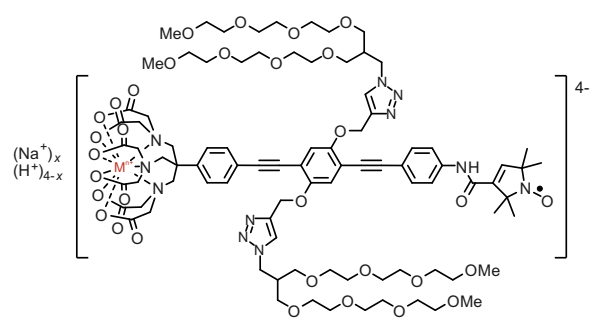

$\left[\mathrm{M}^{\mathrm{n}}\right.$-TAHA]-nitroxide ruler 1

(b)

(c)
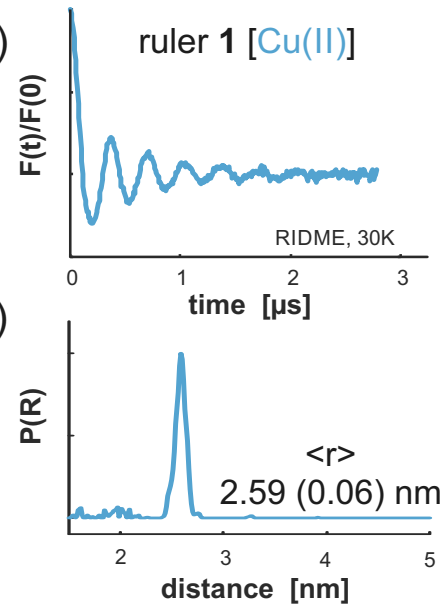

(d)

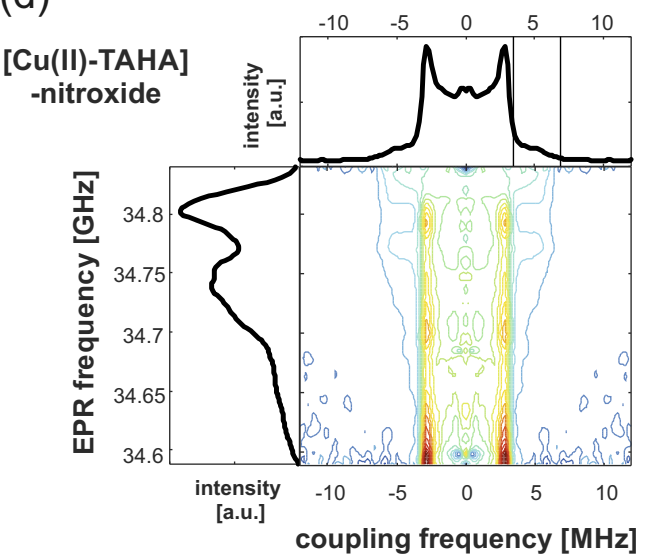

Fig. 6. (a) Chemical structures of two water soluble molecular rulers (examples of $\mathrm{M}^{\mathrm{n}+}$ in color are featured in this article). Different ligands (TAHA in ruler $\mathbf{1}$ and PyMTA in ruler 2) were used. (b) Form factor obtained with ruler $\mathbf{1}$ loaded with $\mathrm{Cu}(I)$ using the RIDME experiment measured at $30 \mathrm{~K}$ with a mixing time of $1.6 \mathrm{~ms}$; (c) distance distribution obtained with ruler $\mathbf{1}$; (d) FT-EPR correlated dipolar coupling pattern obtained with RIDME and frequency-modulated pulses. These coupling patterns contain information about the stiffness and geometry of the molecular ruler. el systems, such as molecular rulers or protein complexes with known structure, has enabled to study the performance of nitroxides, other organic radicals, and metal ion-based spin labels for PDS experiments. The use of spectroscopically orthogonal spin labels can give better confidence and additional information about the extracted distance distributions and, often, improve sensitivity. Currently, we are working on the application of PDS methods and orthogonal spin labelling to investigate the role of protein domains that do not adopt a fixed secondary structure in solution (socalled intrinsically disordered domains). Proteins carrying such domains usually cannot be crystallised and the broadly distributed distances inherent to the conformational ensembles are difficult to resolve by other structural methods. Several human proteins with such domains have been identified as key players in neurodegenerative diseases, for example amyotrophic lateral sclerosis (ALS).[116] PDS methods in combination with spectroscopically orthogonal spin labels are expected to give unique insights into the disordered or only partially ordered states of such proteins.

\section{Acknowledgements}

We thank Anahit Torosyan (ETH Zurich) for help in the preparation of the Rpo4 mutant, Mian Qi (Bielefeld University) for the preparation of the [metal ion-PyMTA] complexes, and Dina Grohmann (University of Regensburg, Germany) for the Rpo4 and Rpo7 plasmids. This work was supported by SNF grant 200020_169057 and DFG (SPP1601; GO555/6-2).

Received: January 24, 2019

[1] C. R. Timmel, J. R. Harmer, 'Structural Information from Spin-Labels and Intrinsic Paramagnetic Centres in the Biosciences', Springer, 2014.

[2] H. J. Steinhoff, Biol. Chem. 2004, 385, 913, DOI: 10.1515/BC.2004.119.

[3] L. J. Berliner, S. S. Eaton, G. R. Eaton, 'Distance Measurements in Biological Systems by EPR', Springer Science \& Business Media, 2006.

[4] D. Abdullin, N. Florin, G. Hagelueken, O. Schiemann, Angew. Chem. 2015, 127, 1847, DOI: 10.1002/ange.201410396.

[5] C. W. M. Kay, H. El Mkami, R. Cammack, R. W. Evans, J. Am. Chem. Soc. 2007, 129, 4868, DOI: 10.1021/ja068966j.
[6] A. V. Astashkin, in 'Methods in Enzymology', Vol. 563, Ed. P. Z. Q. and K. Warncke, Academic Press, 2015, pp. 251

[7] Z. Yang, M. R. Kurpiewski, M. Ji, J. E. Townsend, P. Mehta, L. JenJacobson, S. Saxena, Proc. Natl. Acad. Sci. 2012, 109, E993, DOI: 10.1073/pnas.1200733109.

[8] M. Ezhevskaya, E. Bordignon, Y. Polyhach, L. Moens, S. Dewilde, G. Jeschke, S. V. Doorslaer, Mol. Phys. 2013, 111, 2855, DOI: 10.1080/00268976.2013.813592.

[9] I. Kaminker, M. Bye, N. Mendelman, K. Gislason, S. T. Sigurdsson, D. Goldfarb, Phys. Chem. Chem. Phys. 2015, 17, 15098, DOI: 10.1039/ C5CP01624J.

[10] G. Hanson, 'Metals in Biology - Applications of High-Resolution EPR to Metalloenzymes', Springer, 2018

[11] L. J. Berliner, 'Spin Labeling: Theory and Applications', Academic Press, 2013.

[12] W. L. Hubbell, H. S. Mchaourab, C. Altenbach, M. A. Lietzow, Structure 1996, 4, 779, DOI: 10.1016/S0969-2126(96)00085-8.

[13] W. L. Hubbell, A. Gross, R. Langen, M. A. Lietzow, Curr. Opin. Struct. Biol. 1998, 8, 649, DOI: 10.1016/S0959-440X(98)80158-9.

[14] V. W. Cornish, D. R. Benson, C. A. Altenbach, K. Hideg, W. L. Hubbell, P. G. Schultz, Proc. Natl. Acad. Sci. 1994, 91, 2910, DOI: 10.1073/ pnas.91.8.2910.

[15] E. Bordignon, in 'EPR Spectroscopy', Springer, Berlin, Heidelberg, 2011 pp. 121, DOI: $10.1007 / 128 \_2011 \_243$.

[16] M. M. Haugland, J. E. Lovett, E. A. Anderson, Chem. Soc. Rev. 2018, 47, 668, DOI: 10.1039/C6CS00550K.

[17] P. Roser, M. J. Schmidt, M. Drescher, D. Summerer, Org. Biomol. Chem. 2016, 14,5468 , DOI: $10.1039 / \mathrm{c} 60 b 00473 \mathrm{c}$

[18] S. A. Shelke, S. T. Sigurdsson, Eur. J. Org. Chem. 2012, 2291, DOI: 10.1002/ejoc.201101434.

[19] O. Schiemann, N. Piton, Y. G. Mu, G. Stock, J. W. Engels, T. F. Prisner, J. Am. Chem. Soc. 2004, 126, 5722, DOI: 10.1021/ja0393877.

[20] T. E. Edwards, T. M. Okonogi, B. H. Robinson, S. T. Sigurdsson, J. Am. Chem. Soc. 2001, 123, 1527, DOI: 10.1021/ja005649i.

[21] N. Piton, Y. Mu, G. Stock, T. F. Prisner, O. Schiemann, J. W. Engels, Nucleic Acids Res. 2007, 35, 3128, DOI: 10.1093/nar/gkm169.

[22] U. Jakobsen, S. A. Shelke, S. Vogel, S. T. Sigurdsson, J. Am. Chem. Soc. 2010, 132, 10424, DOI: 10.1021/ja102797k.

[23] S. Obeid, M. Yulikov, G. Jeschke, A. Marx, Angew. Chem. Int. Ed. 2008 47, 6782, DOI: 10.1002/anie.200802314.

[24] G. Y. Shevelev, O. A. Krumkacheva, A. A. Lomzov, A. A. Kuzhelev, O. Y. Rogozhnikova, D. V. Trukhin, T. I. Troitskaya, V. M. Tormyshev, M. V. Fedin, D. V. Pyshnyi, E. G. Bagryanskaya, J. Am. Chem. Soc. 2014, 136, 9874, DOI: 10.1021/ja505122n. 
[25] P. Z. Qin, I. S. Haworth, Q. Cai, A. K. Kusnetzow, G. P. G. Grant, E. A. Price, G. Z. Sowa, A. Popova, B. Herreros, H. He, Nat. Protoc. 2007, 2 , 2354, DOI: $10.1038 /$ nprot.2007.308

[26] G. Sicoli, F. Wachowius, M. Bennati, C. Hoebartner, Angew. Chem. Int. Ed. 2010, 49, 6443, DOI: 10.1002/anie.201000713.

[27] V. Singh, M. Azarkh, T. E. Exner, J. S. Hartig, M. Drescher, Angew. Chem. Int. Ed. 2009, 48, 9728, DOI: 10.1002/anie.200902146.

[28] O. Schiemann, in 'Methods in Enzymology', Vol. 469, Academic Press, 2009, pp. 329, DOI: 10.1016/S0076-6879(09)69016-9.

[29] O. S. Fedorova, Y. D. Tsvetkov, Acta Naturae 2013, 5, 9.

[30] G. Jeschke, V. Chechik, P. Ionita, A. Godt, H. Zimmermann, J. Banham, C. R. Timmel, D. Hilger, H. Jung, Appl. Magn. Reson. 2006, 30, 473, DOI: 10.1007/BF03166213.

[31] Software, http://www.epr.ethz.ch/software.html, accessed March 12, 2019.

[32] A. A. Kuzhelev, O. A. Krumkacheva, G. Y. Shevelev, M. Yulikov, M. V. Fedin, E. G. Bagryanskaya, Phys. Chem. Chem. Phys. 2018, 20, 10224, DOI: 10.1039/C8CP01093E.

[33] I. Dhimitruka, M. Velayutham, A. A. Bobko, V. V. Khramtsov, F. A Villamena, C. M. Hadad, J. L. Zweier, Bioorg. Med. Chem. Lett. 2007, 17, 6801, DOI: 10.1016/j.bmcl.2007.10.030.

[34] D. Akhmetzyanov, P. Schöps, A. Marko, N. C. Kunjir, S. T. Sigurdsson, T. F. Prisner, Phys. Chem. Chem. Phys. 2015, 17, 24446, DOI: 10.1039/ c5cp03671b

[35] J. J. Jassoy, A. Berndhäuser, F. Duthie, S. P. Kühn, G. Hagelueken, O. Schiemann, Angew. Chem. Int. Ed. 2017, 56, 177, DOI: 10.1002/ anie. 201609085.

[36] L. Garbuio, K. Zimmermann, D. Häussinger, M. Yulikov, J. Magn. Reson. 2015, 259, 163, DOI: 10.1016/j.jmr.2015.08.009.

[37] D. Goldfarb, Phys. Chem. Chem. Phys. 2014, 16, 9685, DOI: 10.1039/ C3CP53822B.

[38] T. F. Cunningham, M. D. Shannon, M. R. Putterman, R. J. Arachchige, I. Sengupta, M. Gao, C. P. Jaroniec, S. Saxena, J. Phys. Chem. B 2015, 119, 2839, DOI: $10.1021 /$ jp5103143.

[39] V. Brumas, N. Alliey, G. Berthon, J. Inorg. Biochem. 1993, 52, 287.

[40] M. Qi, A. Groß, G. Jeschke, A. Godt, M. Drescher, J. Am. Chem. Soc. 2014, 136, 15366, DOI: 10.1021/ja508274d.

[41] G. Jeschke, G. Panek, A. Godt, A. Bender, H. Paulsen, Appl. Magn. Reson. 2004, 26, 223, DOI: 10.1007/BF03166574.

[42] G. Jeschke, in 'Annual Review of Physical Chemistry, Vol 63', Eds. M. A. Johnson, T. J. Martinez, Annual Reviews, Palo Alto, 2012, pp. 419.

[43] G. Jeschke, Y. Polyhach, Phys. Chem. Chem. Phys. 2007, 9, 1895, DOI: 10.1039/B614920K

[44] Z. Yang, Y. Liu, P. Borbat, J. L. Zweier, J. H. Freed, W. L. Hubbell, J. Am. Chem. Soc. 2012, 134, 9950, DOI: 10.1021/ja303791p.

[45] Y.-W. Chiang, P. P. Borbat, J. H. Freed, J. Magn. Reson. 2005, 172, 279 DOI: 10.1016/j.jmr.2004.10.012.

[46] T. H. Edwards, S. Stoll, J. Magn. Reson. 2018, 288, 58, DOI: 10.1016/j. jmr.2018.01.021.

[47] S. G. Worswick, J. A. Spencer, G. Jeschke, I. Kuprov, Sci. Adv. 2018, 4, eaat5218, DOI: 10.1126/sciadv.aat5218.

[48] S. Rein, P. Lewe, S. L. Andrade, S. Kacprzak, S. Weber, J. Magn. Reson. 2018, 295, 17, DOI: 10.1016/j.jmr.2018.07.015.

[49] S. Bleicken, G. Jeschke, C. Stegmueller, R. Salvador-Gallego, A. J. García-Sáez, E. Bordignon, Mol. Cell 2014, 56, 496, DOI: 10.1016/j.molcel.2014.09.022.

[50] L. Columbus, W. L. Hubbell, Trends Biochem. Sci. 2002, 27, 288, DOI: 10.1016/S0968-0004(02)02095-9.

[51] B. Joseph, V. M. Korkhov, M. Yulikov, G. Jeschke, E. Bordignon, J. Biol. Chem. 2013, jbc. M113.512178, DOI: 10.1074/jbc.M113.512178.

[52] I. Hänelt, D. Wunnicke, E. Bordignon, H.-J. Steinhoff, D. J. Slotboom, Nat. Struct. Mol. Biol. 2013, 20, 210, DOI: 10.1038/nsmb.2471.

[53] E. R. Georgieva, P. P. Borbat, C. Ginter, J. H. Freed, O. Boudker, Nat. Struct. Mol. Biol. 2013, 20, 215, DOI: 10.1038/nsmb.2494.

[54] O. Duss, M. Yulikov, G. Jeschke, F. H.-T. Allain, Nat. Commun. 2014, 5, 3669, DOI: $10.1038 /$ ncomms4669.

[55] C. Gmeiner, G. Dorn, F. H. T. Allain, G. Jeschke, M. Yulikov, Phys. Chem. Chem. Phys. 2017, 19, 28360, DOI: 10.1039/c7cp05822e.

[56] N. C. Kunjir, G. W. Reginsson, O. Schiemann, S. T. Sigurdsson, Phys. Chem. Chem. Phys. 2013, 15, 19673, DOI: 10.1039/c3cp52789a.

[57] S. Saxena, J. H. Freed, J. Chem. Phys. 1997, 107, 1317, DOI: 10.1063/1.474490

[58] J. S. Becker, S. Saxena, Chem. Phys. Lett. 2005, 414, 248, DOI: 10.1016/j. cplett.2005.08.072

[59] G. Jeschke, M. Pannier, A. Godt, H. W. Spiess, Chem. Phys. Lett. 2000, 331, 243, DOI: 10.1016/S0009-2614(00)01171-4

[60] G. Jeschke, in 'EMagRes', John Wiley \& Sons, Ltd, 2007, DOI: 10.1002/9780470034590.emrstm1518

[61] P. P. Borbat, J. H. Freed, in 'Methods in Enzymology', Vol. 423, Eds. M. I. Simon, B. R. Crane, A. Crane, Academic Press, 2007, pp. 52, DOI: 10.1016/S0076-6879(07)23003-4.
[62] S. Milikisyants, F. Scarpelli, M. G. Finiguerra, M. Ubbink, M. Huber, J. Magn. Reson. 2009, 201, 48, DOI: 10.1016/j.jmr.2009.08.008.

[63] K. B. Konov, A. A. Knyazev, Y. G. Galyametdinov, N. P. Isaev, L. V. Kulik, Appl. Magn. Reson. 2013, 44, 949, DOI: 10.1007/s00723-013-0464-8.

[64] C. E. Tait, S. Stoll, Phys. Chem. Chem. Phys. 2016, 18, 18470, DOI: 10.1039/C6CP03555H.

[65] F. D. Breitgoff, J. Soetbeer, A. Doll, G. Jeschke, Y. O. Polyhach, Phys. Chem. Chem. Phys. 2017, 19, 15766, DOI: 10.1039/c7cp01488k.

[66] K. M. Salikhov, I. T. Khairuzhdinov, Appl. Magn. Reson. 2015, 46, 67, DOI: $10.1007 / \mathrm{s} 00723-014-0609-4$

[67] A. Doll, G. Jeschke, J. Magn. Reson. 2017, 280, 46, DOI: 10.1016/j. jmr.2017.01.004.

[68] P. E. Spindler, S. J. Glaser, T. E. Skinner, T. F. Prisner, Angew. Chem. Int. Ed. 2013, 52, 3425, DOI: 10.1002/anie.201207777.

[69] A. Doll, G. Jeschke, J. Magn. Reson. 2014, 246, 18, DOI: 10.1016/j. jmr.2014.06.016.

[70] L. Garbuio, E. Bordignon, E. K. Brooks, W. L. Hubbell, G. Jeschke, M Yulikov, J. Phys. Chem. B 2013, 117, 3145, DOI: 10.1021/jp401806g.

[71] M. Yulikov, in 'Electron Paramagnetic Resonance', Vol. 24, Eds. V. Chechik, D. M. Murphy, B. Gilbert, Royal Society Of Chemistry, Cambridge, 2014, pp. 1.

[72] E. Narr, A. Godt, G. Jeschke, Angew. Chem. Int. Ed. 2002, 41, 3907

[73] A. Doll, M. Qi, S. Pribitzer, N. Wili, M. Yulikov, A. Godt, G. Jeschke, Phys. Chem. Chem. Phys. 2015, 17, 7334, DOI: 10.1039/C4CP05893C.

[74] A. Doll, M. Qi, N. Wili, S. Pribitzer, A. Godt, G. Jeschke, J. Magn. Reson. 2015, 259, 153, DOI: 10.1016/j.jmr.2015.08.010.

[75] P. Lueders, G. Jeschke, M. Yulikov, J. Phys. Chem. Lett. 2011, 2, 604, DOI: 10.1021/jz200073h.

[76] I. Kaminker, H. Yagi, T. Huber, A. Feintuch, G. Otting, D. Goldfarb, Phys. Chem. Chem. Phys. 2012, 14, 4355, DOI: 10.1039/c2cp40219j.

[77] M. Yulikov, P. Lueders, M. F. Warsi, V. Chechik, G. Jeschke, Phys. Chem. Chem. Phys. 2012, 14, 10732, DOI: 10.1039/C2CP40282C.

[78] I. M. C. van Amsterdam, M. Ubbink, G. W. Canters, M. Huber, Angew. Chem. Int. Ed. 2003, 42, 62, DOI: 10.1002/anie.200390053.

[79] B. E. Bode, J. Plackmeyer, T. F. Prisner, O. Schiemann, J. Phys. Chem. A 2008, 112, 5064, DOI: 10.1021/jp710504k

[80] B. E. Bode, J. Plackmeyer, M. Bolte, T. F. Prisner, O. Schiemann, J. Organomet. Chem. 2009, 694, 1172, DOI: 10.1016/j.jorganchem.2008.11.029.

[81] Z. Yang, J. Becker, S. Saxena, J. Magn. Reson. 2007, 188, 337, DOI: 10.1016/j.jmr.2007.08.006.

[82] A. Meyer, D. Abdullin, G. Schnakenburg, O. Schiemann, Phys. Chem. Chem. Phys. 2016, 18, 9262, DOI: 10.1039/C5CP07621H.

[83] A. M. Bowen, C. E. Tait, C. R. Timmel, J. R. Harmer, in 'Structural Information from Spin-Labels and Intrinsic Paramagnetic Centres in the Biosciences', Springer, Berlin, Heidelberg, 2013, pp. 283, DOI: 10.1007/430_2013_115.

[84] A. Giannoulis, C. L. Motion, M. Oranges, M. Buhl, G. M. Smith, B. E. Bode, Phys. Chem. Chem. Phys. 2018, 20, 2151, DOI: 10.1039/ c7cp07248a.

[85] K. Keller, V. Mertens, M. Qi, A. I. Nalepa, A. Godt, A. Savitsky, G. Jeschke, M. Yulikov, Phys. Chem. Chem. Phys. 2017, 19, 17856, DOI: 10.1039/c7cp01524k.

[86] K. Keller, M. Zalibera, M. Qi, V. Koch, J. Wegner, H. Hintz, A. Godt, G. Jeschke, A. Savitsky, M. Yulikov, Phys. Chem. Chem. Phys. 2016, 18, 25120, DOI: $10.1039 / \mathrm{c} 6 \mathrm{cp} 04884 \mathrm{f}$.

[87] K. Keller, T. Wiegand, R. Cadalbert, B. H. Meier, A. Böckmann, G. Jeschke, M. Yulikov, Chimia 2018, 72, 216, DOI: 10.2533/chimia.2018.216.

[88] D. Abdullin, F. Duthie, A. Meyer, E. S. Mueller, G. Hagelueken, O. Schiemann, J. Phys. Chem. B 2015, 119, 13534, DOI: 10.1021/acs.jpcb.5b02118.

[89] K. Ackermann, C. Pliotas, S. Valera, J. H. Naismith, B. E. Bode, Biophys J. 2017, 113, 1968, DOI: 10.1016/j.bpj.2017.09.005.

[90] P. Lueders, H. Jäger, M. A. Hemminga, G. Jeschke, M. Yulikov, J. Phys. Chem. B 2013, 117, 2061, DOI: 10.1021/jp311287t.

[91] M. Azarkh, O. Okle, V. Singh, I. T. Seemann, J. S. Hartig, D. R. Dietrich, M Drescher, ChemBioChem 2011, 12, 1992, DOI: 10.1002/cbic.201100281.

[92] R. Hänsel, L. M. Luh, I. Corbeski, L. Trantirek, V. Dötsch, Angew. Chem. Int. Ed. 2014, 53, 10300, DOI: 10.1002/anie.201311320.

[93] J. Cattani, V. Subramaniam, M. Drescher, Phys. Chem. Chem. Phys. 2017, 19, 18147, DOI: 10.1039/c7cp03432f.

[94] A. Martorana, G. Bellapadrona, A. Feintuch, E. Di Gregorio, S. Aime, D. Goldfarb, J. Am. Chem. Soc. 2014, 136, 13458, DOI: 10.1021/ja5079392.

[95] Y. Yang, F. Yang, Y.-J. Gong, T. Bahrenberg, A. Feintuch, X.-C. Su, D. Goldfarb, J. Phys. Chem. Lett. 2018, 9, 6119, DOI: 10.1021/acs.jpclett.8b02663.

[96] A. P. Jagtap, I. Krstic, N. C. Kunjir, R. Hänsel, T. F. Prisner, S. T. Sigurdsson, Free Radic. Res. 2015, 49, 78, DOI: 10.3109/10715762.2014.979409.

[97] I. A. Kirilyuk, Y. F. Polienko, O. A. Krumkacheva, R. K. Strizhakov, Y. V. Gatilov, I. A. Grigor'ev, E. G. Bagryanskaya, J. Org. Chem. 2012, 77, 8016, DOI: $10.1021 /$ jo301235j. 
[98] G. Karthikeyan, A. Bonucci, G. Casano, G. Gerbaud, S. Abel, V. Thomé, L. Kodjabachian, A. Magalon, B. Guigliarelli, V. Belle, O. Ouari, E. Mileo, Angew. Chem. 2018, 130, 1380, DOI: 10.1002/ange.201710184.

[99] J. T. Paletta, M. Pink, B. Foley, S. Rajca, A. Rajca, Org. Lett. 2012, 14, 5322, DOI: 10.1021/o1302506f.

[100] Y. Wang, J. T. Paletta, K. Berg, E. Reinhart, S. Rajca, A. Rajca, Org. Lett. 2014, 16, 5298, DOI: $10.1021 / \mathrm{ol} 502449$ r

[101] Z. Wu, A. Feintuch, A. Collauto, L. A. Adams, L. Aurelio, B. Graham, G. Otting, D. Goldfarb, J. Phys. Chem. Lett. 2017, 8, 5277, DOI: 10.1021/acs. jpclett.7b01739.

[102] T. Maly, F. MacMillan, K. Zwicker, N. Kashani-Poor, U. Brandt, T. F. Prisner, Biochemistry 2004, 43, 3969, DOI: 10.1021/bi035865e.

[103] J. Andrecka, R. Lewis, F. Brückner, E. Lehmann, P. Cramer, J. Michaelis, Proc. Natl. Acad. Sci. 2008, 105, 135, DOI: 10.1073/pnas.0703815105.

[104] A. Hirtreiter, D. Grohmann, F. Werner, Nucleic Acids Res. 2010, 38, 585, DOI: $10.1093 /$ nar/gkp928.

[105] D. Grohmann, A. Hirtreiter, F. Werner, Biochem. J. 2009, 421, 339, DOI: 10.1042/BJ20090782.

[106] H. Meka, F. Werner, S. C. Cordell, S. Onesti, P. Brick, Nucleic Acids Res. 2005, 33, 6435, DOI: 10.1093/nar/gki945.

[107] D. Klose, J. P. Klare, D. Grohmann, C. W. M. Kay, F. Werner, H.-J. Steinhoff, PLOS ONE 2012, 7, e39492, DOI: 10.1371/journal.pone.0039492.
[108] S. Pribitzer, M. Sajid, M. Hülsmann, A. Godt, G. Jeschke, J. Magn. Reson. 2017, 282, 119, DOI: 10.1016/j.jmr.2017.07.012.

[109] S. Pribitzer, L. Fábregas Ibáñez, C. Gmeiner, I. Ritsch, D. Klose, M. Sajid, M. Hülsmann, A. Godt, G. Jeschke, Appl. Magn. Reson. 2018, 49, 1253, DOI: 10.1007/s00723-018-1051-9.

[110] Y. Polyhach, E. Bordignon, G. Jeschke, Phys. Chem. Chem. Phys. 2011, 13, 2356, DOI: $10.1039 / \mathrm{COCP} 01865 \mathrm{~A}$.

[111] T. von Hagens, Y. Polyhach, M. Sajid, A. Godt, G. Jeschke, Phys. Chem. Chem. Phys. 2013, 15, 5854, DOI: 10.1039/C3CP44462G.

[112] G. Jeschke, M. Sajid, M. Schulte, A. Godt, Phys. Chem. Chem. Phys. 2009, 11, 6580, DOI: 10.1039/B905724B.

[113] C. Gmeiner, D. Klose, E. Mileo, V. Belle, S. R. A. Marque, G. Dorn, F. H. T. Allain, B. Guigliarelli, G. Jeschke, M. Yulikov, J. Phys. Chem. Lett. 2017, 8, 4852, DOI: 10.1021/acs.jpclett.7b02220.

[114] L. Garbuio, B. Lewandowski, P. Wilhelm, L. Ziegler, M. Yulikov, H. Wennemers, G. Jeschke, Chem. - Eur. J. 2015, 21, 10747, DOI: 10.1002/ chem.201501190.

[115] A. Giannoulis, M. Oranges, B. E. Bode, Chem. Phys. Chem. 2017, 18 2318, DOI: $10.1002 / \mathrm{cphc} .201700666$

[116] M. D. Purice, J. P. Taylor, Front. Neurosci. 2018, 12, DOI: 10.3389/ fnins.2018.00326. 